Instructions for authors, subscriptions and further details:

http://redimat.hipatiapress.com

\title{
Enseñanza de Geometría Sintética a Futuros Profesores. El caso de la Universidad Nacional de Rosario
}

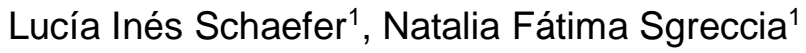

1) Universidad Nacional de Rosario. Argentina

Date of publication: Junio $24^{\text {th }}, 2018$

Edition period: Junio 2018-Octubre 2018

To cite this article: Schaefer, L.I., \& Sgreccia, N.F. (2018). Enseñanza de geometría sintética a futuros profesores. El caso de la Universidad Nacional de Rosario. REDIMAT - Journal of Research in Mathematics Education, 7(2), 134-161. doi: 10.4471/redimat.2018.2559

To link this article: http://dx.doi.org/10.4471/redimat.2018.2559

PLEASE SCROLL DOWN FOR ARTICLE

The terms and conditions of use are related to the Open Journal System and to Creative Commons Attribution License (CCAL). 


\section{Teaching of Synthetic Geometry to Future Teachers. The case of the National University of Rosario}

Lucía Inés Schaefer

Universidad Nacional de

Rosario
Natalia Fátima Sgreccia

Universidad Nacional de

Rosario

(Received: 10 February 2017; Accepted: 22 April 2018;Published: 24 June 2018)

\section{Abstract}

The general aim of this article is to know about the promotion of knowledge building related to Synthetic Geometry in the initial training of teachers in Mathematics. It is expected to identify, describe and come up with the teaching practices carried out in the first year of the Mathematics Teacher Training career in the National University of Rosario (Argentina). The mathematical knowledge for teaching (MKT) of the Michigan group is taken as a reference. The research has a qualitative approach and is empirical, in its natural context and with descriptive scope. The categories of analysis correspond to the six sub-domains of the MKT. Among the data gathering techniques is the observation of classes and for the data processing the content analysis is applied, starting on the recognition of regularities in registers. Among the main findings is the importance given by the teacher to the students' building of concepts using different strategies. It is concluded that although the teacher makes an approach to the school version of contents, it is not explicitly done to the teachers-to-be.

Keywords: teacher training - MKT - synthetic geometry 


\section{Enseñanza de Geometría Sintética a Futuros Profesores. El caso de la Universidad Nacional de Rosario}

Lucía Inés Schaefer

Universidad Nacional de

Rosario
Natalia Fátima Sgreccia Universidad Nacional de Rosario

(Recibido: 10 Febrero 2017; Aceptado: 22 Abril 2018;Publicado: 24 Junio 2018)

\section{Resumen}

El presente artículo tiene como objetivo general conocer acerca de la promoción de construcción de conocimientos relativos a Geometría Sintética en la formación inicial de profesores en Matemática. Se espera identificar, describir y conceptualizar las prácticas de enseñanza llevadas a cabo en el primer año del Profesorado en Matemática de la Universidad Nacional de Rosario (Argentina). Se toma como referencia el conocimiento matemático para la enseñanza (MKT) del grupo Michigan. La investigación tiene un enfoque cualitativo y es de tipo empírica, en su contexto natural y con alcance descriptivo. Las categorías de análisis se corresponden con los seis subdominios del MKT. Entre las técnicas de recolección de información se encuentra la observación de clases y para su procesamiento se aplica el análisis de contenido, a partir de reconocer regularidades en los registros. Entre los principales hallazgos se encuentra la importancia otorgada por el docente a la construcción de los conceptos por parte de los estudiantes utilizando distintas estrategias. Se concluye que si bien el docente realiza un acercamiento a la versión escolar de los contenidos, no lo hace de forma explícita a los futuros profesores.

Palabras clave: formación de profesores - MKT - geometría sintética

2018 Hipatia Press

ISSN: 2014-3621

DOI: $10.4471 /$ redimat.2018.2559

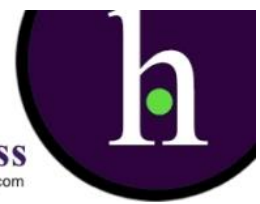




\section{Schaefer \& Sgreccia - Enseñanza de la geometría sintética}

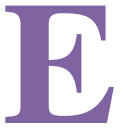

1 presente artículo se enmarca en un plan de investigación denominado "La Geometría Sintética en la Formación del Profesor en Matemática: el caso de la Universidad Nacional de Rosario", correspondiente a una Beca de Estímulo a las Vocaciones Científicas, otorgada por el Consejo Interuniversitario Nacional (Argentina) durante septiembre 2015-2016, cuyo propósito general es conocer acerca de las prácticas de enseñanza para la construcción de conocimientos de Geometría Sintética en la formación inicial de profesores en Matemática. Toma como caso a la carrera Profesorado en Matemática (PM) de la Universidad Nacional de Rosario.

Como referentes teórico-metodológicos se adoptan los modelos propuestos por los equipos liderados por Deborah Ball de la Universidad de Michigan (Estados Unidos) y por César Coll de la Universidad de Barcelona (España). El primero refiere al conocimiento matemático para la enseñanza (MKT) y comprende seis subdominios de conocimiento que un profesor en Matemática debería poseer: común del contenido, en el horizonte matemático, especializado del contenido, del contenido y de los alumnos, del contenido y de la enseñanza, del contenido y del currículum.

En segundo término, del estudio de mecanismos de influencia educativa se considera especialmente lo relativo a la detección de configuraciones de mensajes en sesiones de clase.

Entre los objetivos específicos se destaca identificar, describir y conceptualizar las prácticas de enseñanza de Geometría Sintética desarrolladas en el primer año de la carrera, y caracterizar las acciones docentes que promueven u obstaculizan las oportunidades de aprendizaje de contenidos geométricos. También se espera reconocer los modos de activación de los dominios del MKT en el desarrollo de las clases y en el material de estudio, identificar momentos fértiles de vigilancia epistémica en la formación de profesores, así como explorar los puntos de vista de docentes y estudiantes acerca de propuestas didácticas alternativas para el abordaje de algunos contenidos de Geometría Sintética.

Gutiérrez (2010) señala que en la actual escuela secundaria la Geometría Sintética está escasamente desarrollada y muchas veces se olvida cómo tratarse durante la formación de profesores. Lo primero imposibilita a los estudiantes conocer otro modo de pensar; respecto a lo segundo, el tipo de experiencias formativas por las cuales transita un futuro profesor es determinante para su desempeño profesional (Ministerio de Educación, 
2010). La investigación sobre el conocimiento de los profesores en Matemática ha surgido en las últimas tres décadas (Ball, 1988; Barrantes y Blanco, 2005), acompañados por una preocupación pública y política como medio para mejorar la enseñanza (Borba, 2006; García, 2005; Gascón, 2002). Poco se sabe acerca de los conocimientos matemáticos para la enseñanza, con importante vacancia en la Geometría Sintética (Sgreccia y Massa, 2012).

La Geometría Sintética se reconoce como uno de los componentes más importantes del currículum escolar de Matemática (Atiyah, 2001). Las razones para su inclusión son múltiples: promover la percepción espacial, la intuición y la visualización, estimular la creatividad, desarrollar habilidades como conjeturar, razonar deductivamente y argumentar de manera lógica en demostraciones, modelizaciones y resolución de problemas. Por otro lado, en los núcleos temáticos básicos a desarrollar en los Profesorados en Matemática Universitarios de Argentina (Consejo Interuniversitario Nacional, 2013), se distingue al área Geometría como ejemplo paradigmático para la enseñanza de una teoría axiomático-deductiva y se subraya su potencial para el desarrollo de la intuición, inducción, visualización, percepción de relaciones, regularidades y propiedades. En particular, la Geometría Sintética es la base estructural de toda una rama dentro de la Matemática y del pensamiento matemático mismo.

Las conjeturas iniciales que orientan este trabajo de investigación son dos: la versión de la Geometría Sintética estudiada en el Profesorado en Matemática dista considerablemente de una versión escolar relativamente propicia de procesos constructivos en la escuela secundaria; y el conocimiento especializado del contenido de Geometría Sintética, requerido para la enseñanza, queda relegado a la construcción, individual y sin vigilancia epistémica, del estudiante. Para fundamentar o rechazar estas conjeturas, se procedió al análisis de relatos de observaciones, a la aplicación de cuestionarios a los alumnos y al análisis del apunte de la asignatura. En esta ocasión se presentan los hallazgos relativos a la primera actividad señalada.

\section{Algunos Estudios Relacionados}

En torno a los trabajos realizados por Ball, surgen distintas investigaciones con el objetivo de describir de manera profunda el conocimiento que 
necesita un profesor. Santana y Climent (2015) cuestionan la poca claridad en los límites del conocimiento común del contenido propuesto por el grupo de Michigan, y presentan nuevos subdominios dentro del conocimiento especializado del contenido. Basan su estudio en el análisis de clases donde se trabaja con software matemático, para lo cual determinan algunos indicadores agrupados por bloques. De la misma manera, Escudero, Carrillo y Flores (2015) buscan superar algunas dificultades por ellos identificadas en el trabajo del equipo estadounidense y presentan el modelo del conocimiento especializado del profesor en matemática (MTSK), en el cual confluyen distintos conocimientos matemáticos y didácticos, y las concepciones y creencias del docente. También ofrecen un ejemplo de cómo analizar las clases "desempaquetando" las acciones docentes desde los lineamientos del modelo presentado.

En cuanto al análisis de subdominios particulares del MKT, De Gamboa, Badillo y Ribeiro (2015) se centran en el conocimiento en el horizonte matemático (HCK), considerando que el mismo refiere al conocimiento del profesor que da forma y permite conectar otros tipos de conocimiento en la práctica. Sostienen que debe ser estudiado tanto desde la perspectiva de la matemática como desde la pedagogía y el currículo. En su investigación, analizan y ejemplifican diversas situaciones, lo que colabora con la comprensión del subdominio mencionado. Otros autores, como Sgreccia y Massa (2012), estudian temas específicos de geometría utilizando el conocimiento especializado del contenido como herramienta para el análisis. En particular, en el trabajado señalado se busca conocer acerca de la competencia espacial y de la didáctica de la geometría $3 \mathrm{~d}$ en la formación de profesores. A partir de cuestionarios abiertos aplicados a estudiantes avanzados y egresados recientes, concluyen sobre la importancia de que todo profesor tenga en mente posibles orientaciones para las dificultades de los alumnos, destacando esto como un componente fundamental en el conocimiento para la enseñanza de la matemática. Godino, Gonzato, Contreras, Estepa y Díaz-Batanero (2016) también abarcan las habilidades espaciales de futuros maestros en España y concluyen sobre la imperiosa necesidad de poner mayor énfasis en los temas de didáctica de la matemática y de proporcionar una mejor cobertura de los temas de las matemáticas escolares en la formación de los docentes.

Así como Escudero et al. (2015) consideran las concepciones y creencias como un componente importante del conocimiento del profesor, 
Barrantes y Blanco (2005) también lo hacen y analizan cómo estas concepciones implícitas originadas en la propia escolaridad del docente influyen en la enseñanza y el aprendizaje de la Geometría. Señalan una disociación entre dos culturas diferentes: la clásica que vivieron como alumnos y la actual. Por esta razón, revaloran la formación inicial de los profesores como punto clave donde abordar los recuerdos y expectativas de los mismos. Yang (2012) también trabaja sobre esta temática y destaca la importancia de detectar las creencias y actitudes hacia la disciplina en la formación inicial, para poder redireccionar aquellas negativas y mejorar la futura práctica docente. Además, Taylan y Da Ponte (2016) destacan que si en esta formación inicial de profesores participan docentes que recorren distintos roles (maestro, investigador, formador), se obtienen ideas relativas a desarrollar el conocimiento necesario para la enseñanza y analizarlo, con el fin de facilitar el aprendizaje de los futuros profesores. En su artículo estudian cómo se transforma el conocimiento pedagógico del contenido (PCK) de los formadores, propuesto por Shulman, a partir de la interacción con estudiantes y su posterior reflexión.

Las investigaciones reportadas aportan a la comprensión del modelo del MKT y ofrecen herramientas útiles para el análisis de las clases observadas. Por otro lado, autores como Corica y Marin (2014) y Acosta, Mejía y Rodríguez (2013) proponen una estructura distinta para las clases, basada en la noción de actividades de estudio e investigación (en el marco de la Teoría Antropológica de Chevallard) en las cuales los alumnos, guiados por el profesor, estudian y reconstruyen alguna organización matemática. En ella se prioriza la construcción de los conceptos por parte de los estudiantes a partir de una situación inicial. Esto proporciona aspectos a tener en cuenta en la elaboración de una propuesta didáctica para la enseñanza de la Geometría, a partir del análisis realizado.

\section{Encuadre Teórico}

En 1983, Shulman trata en una de sus conferencias "el paradigma perdido" de la investigación de la enseñanza y el contenido del profesor, otorgando con ello especial atención al rol del contenido de una disciplina en su enseñanza. Propone un dominio especial del conocimiento del profesor, denominado conocimiento pedagógico del contenido, y lo caracteriza como un puente entre el conocimiento del contenido y de la práctica de la 


\section{Schaefer \& Sgreccia - Enseñanza de la geometría sintética}

enseñanza. De esta manera, abre una importante rama de investigación seguida por numerosos autores.

Ball, Thames y Phelps (2008) presentan una teoría basada en la práctica, la del MKT, considerado como el conocimiento matemático necesario para llevar a cabo el trabajo de la enseñanza de esta disciplina. A partir del análisis de clases grabadas de Educación Primaria en Estados Unidos, concluyen que el conocimiento matemático necesario para la enseñanza es multidimensional y que requiere el detalle en numerosos aspectos innecesarios para cualquiera otra área. Es decir, un docente debe conocer más y de manera diferente la matemática.

Los autores resaltan que su punto de análisis no es lo que los docentes necesitan enseñarles a sus alumnos, sino qué es lo que los docentes deben conocer para sostener tal enseñanza. Ofrecen un camino adicional para construir el puente mencionado por Shulman, incluyendo las habilidades y los hábitos de razonamiento al conocimiento necesario por los profesores. Sostienen como hipótesis que es posible reconocer subdominios (los cuatro que se presentan a continuación), tanto en el conocimiento de la disciplina (los dos primeros) como en el conocimiento pedagógico del contenido (los dos últimos).

- Conocimiento común del contenido (CCK): es el conocimiento matemático y las habilidades que pueden ser utilizadas en un marco distinto al de la enseñanza, es decir, por cualquier persona que haya estudiado matemática. Los profesores deben ser capaces de resolver todo aquello que le plantean a sus alumnos, de detectar cuándo algo no es correcto, de utilizar la notación adecuada. Los autores destacan, además, que con "común" no refieren a que todos cuentan con este conocimiento sino que puede ser utilizado en distintos ámbitos, no necesariamente vinculados con la enseñanza.

- Conocimiento especializado del contenido (SCK): es el conocimiento matemático propio de la enseñanza, es decir, que no es necesario en otros ámbitos distintos a este. El docente debe comprender distintas interpretaciones de un mismo concepto, dado que la enseñanza de la disciplina implica volver visibles y enseñables determinadas características del contenido para los alumnos. La diferencia con el $\mathrm{CCK}$ es que este refiere al conocimiento puesto en juego al resolver un problema, tanto por un matemático como por cualquier otro sujeto que esté capacitado, mientras que el SCK atiende a ordenar 
las secuencias con que podrían desarrollarse distintos aspectos de un contenido específico.

- Conocimiento del contenido y de los alumnos (KCS): es el conocimiento que combina el saber sobre los alumnos y el saber sobre la matemática. El docente debe poder anticipar qué pensarán sus alumnos, qué dificultades tendrán, qué encontrarán interesante y qué no, así como escuchar e interpretar las ideas y pensamientos incompletos de los estudiantes.

- Conocimiento del contenido y de la enseñanza (KCT): combina el conocimiento sobre la enseñanza y el conocimiento de la matemática. El docente debe conocer cuestiones para el diseño y planificación de las clases, la secuenciación de contenidos, la identificación de métodos y procedimientos adecuados para cada situación. En una clase el docente debe decidir cuándo aprovechar las ideas de los alumnos, cuándo efectuar nuevas preguntas o cuándo realizar una pausa para aclarar algo.

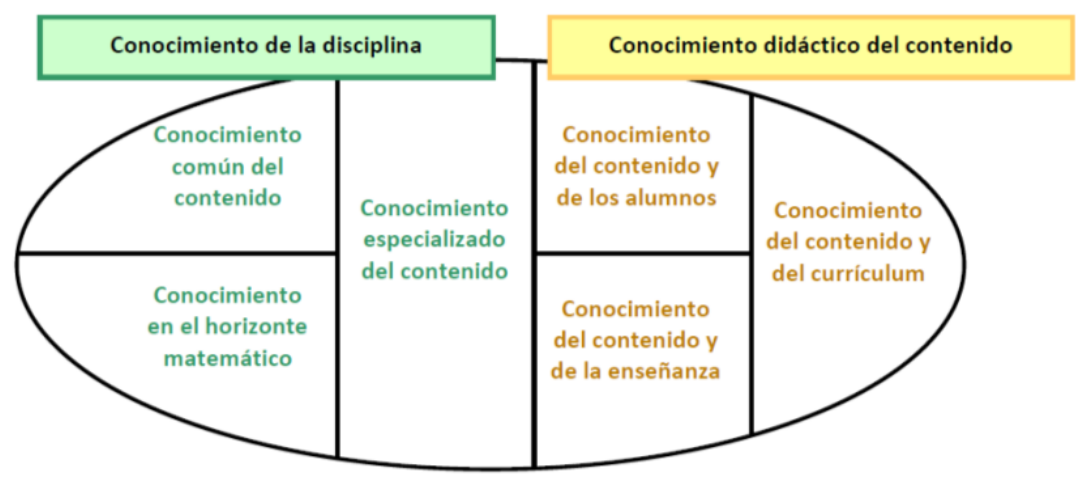

Figura 1. Dominios y subdominios del MKT

Para visualizar estas ideas, los autores proponen el diagrama de la Fig. 1, en el cual se observa cómo el conocimiento del currículum propuesto por Shulman es colocado dentro del conocimiento pedagógico del contenido. Sin embargo afirman no estar seguros si podría ser parte del KCT o si podría ser transversal a varias categorías. Además incluyen una tercera categoría en el conocimiento de la disciplina a la cual denominan 
conocimiento en el horizonte matemático (HCK), que incluye la capacidad de realizar conexiones con contenidos matemáticos futuros y de tomar decisiones sobre cómo tratar ciertos temas en cada situación. Queda así constituido el modelo con los seis subdominios.

Los autores concluyen que aquel profesor que no conozca bien la materia no tendrá el conocimiento necesario para enseñar el contenido, pero conocer bien un tema no es suficiente para la enseñanza. Los docentes necesitan conocer la matemática de tal manera que sean capaces de dotar de sentido el trabajo de los estudiantes y de elegir la mejor forma de representar el contenido.

Por otro lado, Coll, Colomina, Onrubia y Rochera (1992) focalizan su atención en la enseñanza. Pretenden comprender cómo los alumnos aprenden determinados contenidos como consecuencia de la influencia educativa que ejerce el profesor sobre ellos. Con este fin proponen identificar aquellos mecanismos mediante los cuales una persona incide sobre otra ayudándole a construir un sistema de significados compartidos referidos a algún tema. Uno de estos mecanismos que sostienen como hipótesis es la cesión y traspaso progresivos de la responsabilidad y control en el aprendizaje.

Los autores toman el concepto de interactividad como una forma de organización de la actividad conjunta entre docente y alumno, lo cual implica tener en cuenta la dimensión temporal de los procesos de enseñanza y aprendizaje, el contenido y/o tarea en torno al cual se articula la actividad conjunta, y ciertas unidades de análisis. Respecto a estas últimas, consideran las secuencias didácticas, las cuales son diseñadas respetando su contexto natural. Dado que esta última es una unidad de análisis global, surge otra de menor amplitud denominada sesión de trabajo y, como en ocasiones esta parcelación no aporta demasiada información, se determina otra unidad de análisis más pequeña llamada segmento de interactividad. Estos segmentos de interactividad se definen por el conjunto de actuaciones esperadas o esperables y aceptadas o aceptables de los participantes. Para identificarlos se siguen dos criterios: la unidad temática o de contenido y el patrón de comportamientos dominantes. Sin embargo no permiten profundizar en los procesos de construcción de significados, por lo cual se presenta una nueva unidad de análisis, de naturaleza semiótica, denominada mensajes. Esta es la unidad mínima y refiere a la expresión de uno de los 
participantes que tiene sentido por sí misma y que no puede descomponerse en unidades más elementales sin perder el significado que transmite.

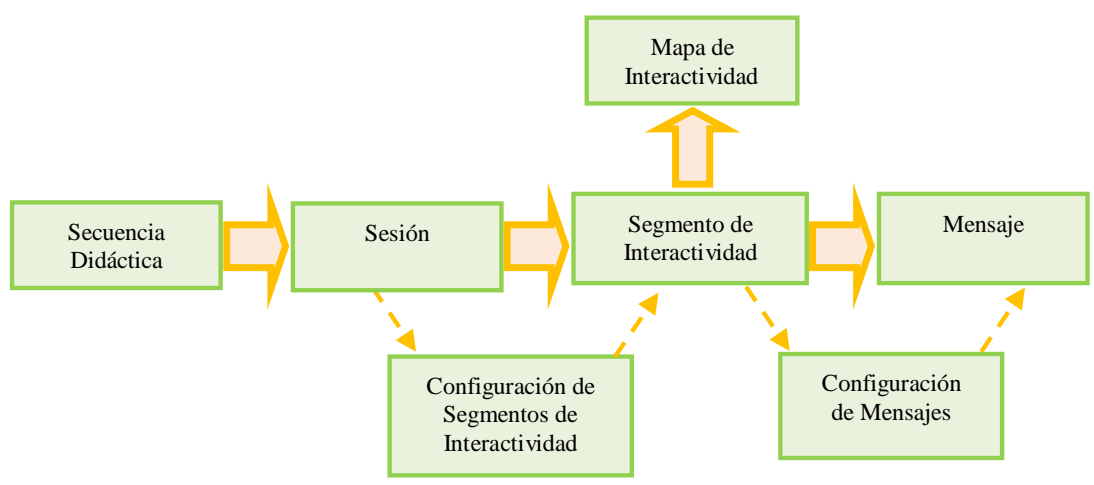

Figura 2. Esquema de segmentación del contenido de las clases

Junto a las cuatro unidades básicas de análisis señaladas, los autores muestran la existencia de una configuración de segmentos de interactividad que abarca varios segmentos de interactividad relacionados entre sí. También mencionan una unidad de análisis intermedia entre los segmentos de interactividad y los mensajes, llamada configuración de mensajes (CM), referida a agrupaciones de mensajes que transmiten significados que no pueden reducirse a la suma de los significados transmitidos por cada uno de los mensajes que la integran. Estas últimas son las que se toman como unidad de análisis en el presente trabajo. Un esquema que procura representar estas ideas se presenta en la Fig. 2 (Ciccioli y Sgreccia, 2017).

\section{Método}

El caso en estudio es el PM, una de las once carreras de grado de la Facultad de Ciencias Exactas, Ingeniería y Agrimensura de la Universidad Nacional de Rosario (Argentina), cuyo plan de estudios es de cuatro años de duración. En particular, Geometría I es una de las cuatro asignaturas anuales de primer año, con siete horas reloj semanales de cursado, que se desarrolla en común con la carrera Licenciatura en Matemática, durante 30 
semanas comprendidas entre principios de marzo a fines de junio (primer semestre, donde se cursa la parte relativa a Geometría Sintética) y principios de agosto a fines de noviembre (segundo semestre, en la que se estudia Geometría Analítica).

En el programa vigente de Geometría I se especifica que en ambas partes (Geometría Sintética y Analítica) se estudia tanto el plano como el espacio euclídeo. Además, se reconoce a la Geometría Euclídea como el ámbito propicio para que el alumno que recién se inicia en el aprendizaje profundo de la matemática comience a desarrollar las aptitudes más importantes que necesitará tanto en las asignaturas subsiguientes como en su labor profesional futura. Esto se debe a que en esta asignatura se introduce el primer sistema axiomático donde los teoremas y definiciones se deducen con rigurosidad lógica a partir de estos hasta construir una teoría matemática profunda, lo que constituye la base del quehacer matemático. También, debido a que se introduce el razonamiento abstracto con modelos concretos que los alumnos traen parcialmente incorporados de la escuela secundaria, procurando que la asimilación de nuevos conceptos y de nuevas formas de razonar se realice sobre la base de una experiencia previa. En la materia se presenta además una gran variedad de problemas donde se introduce tanto la modelización de situaciones reales que pueden resolverse con argumentos matemáticos sencillos, así como de problemas teóricos más profundos. Se manifiesta que en todos ellos se pretende estimular la creatividad y la rigurosidad en el razonamiento de los estudiantes.

En particular, la parte de Geometría Sintética comprende ocho unidades temáticas: 1. El espacio geométrico; 2. Figuras planas; 3. Medidas de figuras planas; 4. Más sobre figuras planas; 5. Cuerpos: características y medidas; 6. Semejanza; 7. Trigonometría; 8. Movimientos en el plano. El trabajo realizado en esta investigación se basa en observaciones de ocho clases (que abarcan las unidades temáticas 2 y 3, desarrolladas durante un mes -del 8 de abril al 8 de mayo- y a un mes de comenzado el año lectivo) de la asignatura Geometría I del PM.

Se tomó registro escrito exhaustivo de las mismas con su posterior redacción a modo de relato. En estos relatos se distinguieron los distintos momentos de las clases, siguiendo la idea de CM propuestas por Coll et al. (1992). Después se procedió a identificar en los relatos de observaciones los distintos subdominios del MKT (Ball et al., 2008) y dentro de cada uno de ellos surgieron diversas modalidades. Estas representan el contenido 
concreto relativo a particularidades de cada subdominio que fue posible reconocer y cuya delimitación caracteriza el conocimiento para enseñar Geometría Sintética que pone en juego y promueve el docente del curso (se harán explícitas en la parte correspondiente a resultados). De este modo cada modalidad se asocia a uno o más mensajes (extractos de los relatos) que, si bien pueden estar expresados con palabras distintas, tienen la misma naturaleza semiótica (que es lo que se procura dar a conocer mediante la denominación de la modalidad).

A partir de lo anterior, se elaboró una matriz para cada clase, en la cual se volcaron los momentos de las clases y los dominios que se activaron en cada uno de ellos. Esta síntesis colaboró en el análisis, el cual derivó en un esquema lógico-conceptual que concentró las regularidades halladas. Estas cuestiones resultaron provechosas para la detección de limitaciones y oportunidades en las clases, lo cual constituyó el puntapié para elaborar una propuesta didáctica para el PM. En este caso, las limitaciones refieren a alguna cuestión de la enseñanza que entorpece la compresión de los estudiantes, mientras que con oportunidades se hace alusión a momentos provechosos y útiles que colaboran con la construcción del conocimiento matemático para la enseñanza de la Geometría Sintética de los alumnos que serán futuros docentes.

En cuanto a los participantes de la investigación, se destaca que es el cuarto año consecutivo que el docente se desempeña en la materia, tiene 30 años de edad y casi 10 de antigüedad en docencia universitaria. Posee los títulos de Licenciado y Doctor en Matemática, y desarrolla una carrera como investigador en la disciplina. El curso está formado por 70 alumnos, de los cuales 2 son recursantes (habían cursado la materia el año anterior pero con desempeños no satisfactorios) y las dos terceras partes aproximadamente corresponden a la carrera PM. Si bien en su mayoría son recién egresados de la escuela secundaria (18 años de edad), es posible distinguir algunos de mayor edad (llegando incluso a los 60 años). Acerca de la procedencia, casi todos son de Rosario o de localidades cercanas (de la provincia de Santa Fe, Entre Ríos o norte de Buenos Aires), presentándose algunos casos de extranjeros (Venezuela, Colombia). 
144 Schaefer \& Sgreccia - Enseñanza de la geometría sintética

\section{Resultados}

A partir de los relatos de observaciones de las clases, se procedió a distinguir en ellos tanto las CM de interactividad (Coll et al., 1992) como los subdominios del MKT (Ball et al., 2008).

Tabla 1.

CM de las clases observadas

\begin{tabular}{ll}
\hline Clase & CM \\
\hline & 1. Introducción a la medida de segmentos \\
2. Base axiomática de la medida de segmentos \\
3. Definiciones variadas \\
4. Procedimientos \\
5. Introducción a la medida de ángulos \\
6. Base axiomática de la medida de ángulos \\
7. Definiciones variadas \\
8. Continuación de “Base axiomática de la medida \\
de ángulos" \\
9. Procedimientos \\
10. Definiciones y propiedades a partir de los \\
axiomas \\
11. Cierre de la clase \\
1. Repaso de contenidos \\
2. Construcción y definición de bisectriz \\
3. Definiciones de cuadriláteros \\
4. Definición y propiedades de congruencia de \\
triángulos \\
5. Cierre de la clase \\
1. Repaso de contenidos \\
2. Demostración de lema \\
3. Definiciones y demostración de ángulos entre \\
rectas paralelas \\
4. Demostraciones variadas \\
5. Cierre de la clase \\
1. Repaso de contenidos \\
2. Construcción de criterios de congruencia de \\
triángulos \\
3. Cierre de la clase \\
\hline \multirow{4}{*}{3}
\end{tabular}


Tabla 1. (.../...)

\begin{tabular}{|c|c|}
\hline Clase & $\mathbf{C M}$ \\
\hline \multirow{3}{*}{5} & 1. Repaso de contenidos \\
\hline & $\begin{array}{l}\text { 2. Enunciado, demostración y utilidad de lema y } \\
\text { criterios }\end{array}$ \\
\hline & 3. Cierre de la clase \\
\hline \multirow{9}{*}{6} & 1. Disculpas y motivación a los alumnos \\
\hline & 2. Repaso de contenidos \\
\hline & 3. Resolución de problemas \\
\hline & 4. Demostración de criterios de congruencia de \\
\hline & triángulos \\
\hline & 5. Definición de congruencia de polígonos \\
\hline & 6. Introducción a área \\
\hline & 7. Base teórica de área y demostraciones \\
\hline & 8. Cierre de la clase \\
\hline \multirow{2}{*}{7} & 1. Repaso de contenidos \\
\hline & 2. Demostración de teoremas de área \\
\hline \multirow{3}{*}{8} & 1. Introducción al teorema de Pitágoras \\
\hline & 2. Demostración del teorema de Pitágoras \\
\hline & 3. Cierre de la clase de teoría \\
\hline
\end{tabular}

En la Tabla 1 se presentan las CM identificadas en las ocho clases observadas. Se puede distinguir que, por lo general, las clases comenzaban con un repaso de contenidos, ya sean previos o dados en oportunidades anteriores en la misma asignatura. Otra regularidad es el cierre de la clase, a veces antecedido por demostraciones o definiciones variadas. Cabe destacar que tanto el repaso de contenidos como el cierre de la clase tenían una duración menor a cinco minutos. El desarrollo en sí de las clases (CM intermedias) se caracterizó por lo general mediante la estructura: introducción de conceptos $\rightarrow$ definición $\rightarrow$ demostración $\rightarrow$ procedimientos $\rightarrow$ problemas.

En el proceso de identificación de los subdominios del MKT en los relatos de clases, surgieron diversas modalidades asociadas a ellos, las cuales se presentan en las Tablas 2 a 7 -correspondiéndose con CCK, HCK, $\mathrm{SCK}, \mathrm{KCS}, \mathrm{KCT}$ y KCC respectivamente-. Para cada una se indica su frecuencia de detección en los relatos y se acompaña con un ejemplo, consistente en un extracto de lo acontecido en las clases que procura ilustrar 
el sentido semiótico de la modalidad. Cabe advertir que cuando se emplean comillas se trata de palabras textuales del profesor.

Tabla 2.

Modalidades y extractos relativos a CCK

\begin{tabular}{ll}
\hline Modalidad & Extracto del relato \\
\hline $\begin{array}{l}\text { Formalización } \\
\text { (40) }\end{array}$ & $\begin{array}{l}\text { Explica así el Axioma 13 y luego lo escribe en el } \\
\text { pizarrón }\end{array}$ \\
$\begin{array}{l}\text { Definición (20) } \\
\text { "Bxplicación de } \\
\text { procedimientos }\end{array}$ & $\begin{array}{l}\text { Explica cómo llevar a cabo el transporte de ángulos } \\
(12)\end{array}$ \\
$\begin{array}{l}\text { Introducción de } \\
\text { notación (7) }\end{array}$ & $\begin{array}{l}\text { Aclara que los lados con la misma cantidad de marcas } \\
\text { son congruentes, y lo mismo sucede para los ángulos }\end{array}$ \\
$\begin{array}{l}\text { Verificación de } \\
\text { procedimientos }\end{array}$ & $\begin{array}{l}\text { Luego comprueba, utilizando las herramientas de } \\
\text { GeoGebra, que los ángulos que obtuvieron son iguales }\end{array}$ \\
& $\begin{array}{l}\text { a } \boldsymbol{\alpha}, \text { verificando así que el procedimiento llevado a } \\
\text { cabo es correcto }\end{array}$ \\
\hline
\end{tabular}

En la Tabla 2 se puede advertir que el proceso de formalización se hizo muy presente en las clases, seguido por la definición. En la mayoría de los casos, estos dos procesos los desarrolló en interacción con el grupo-clase. En cuanto a los procedimientos, siempre se llevaron a cabo con su respectiva explicación, lejos de recetas memorísticas, y aproximadamente en la mitad de las ocasiones, se los acompañó con su respectiva verificación. La notación específica que se empleó también se ha ido introduciendo con su razón de ser, sin arbitrariedades.

Como se puede notar en la Tabla 3, el docente efectuó connotaciones hacia los contenidos que se estaban trabajando, ponderándolos desde una perspectiva matemática holística. El contexto histórico fue tenido en cuenta en numerosas oportunidades, principalmente para introducir un tema $\mathrm{y}$ enfatizar la necesidad de su surgimiento. En ocasiones explicitó la razón de ser de las formalizaciones, otorgándoles sentido. También procuró crear cierto grado de conciencia sobre las clasificaciones y denominaciones: cómo pueden ser y hasta dónde abarcan. Algunas veces aludió a conexiones 
con otros ámbitos, no matemáticos o del futuro desempeño laboral de los profesores.

Tabla 3.

Modalidades y extractos relativos a $\mathrm{HCK}$

\begin{tabular}{|c|c|}
\hline Modalidad & Extracto del relato \\
\hline $\begin{array}{l}\text { Connotación hacia } \\
\text { los contenidos (14) }\end{array}$ & $\begin{array}{l}\text { "Este es uno de los temas más importantes de la } \\
\text { materia, de acá a fin de año en todos los ejercicios lo } \\
\text { van a tener que usar junto a semejanza" }\end{array}$ \\
\hline $\begin{array}{l}\text { Contexto histórico } \\
\text { (9) }\end{array}$ & $\begin{array}{l}\text { Comienza así a contar la historia de Pitágoras similar a } \\
\text { la que figura en el apunte. Comenta acerca de los } \\
\text { pitagóricos, agregando luego que en esa época los } \\
\text { números eran distintos }\end{array}$ \\
\hline $\begin{array}{l}\text { Sentido de la } \\
\text { formalización (5) }\end{array}$ & $\begin{array}{l}\text { "La regla es la representación mecánica del axioma } 2 \text { o } \\
3 \text {, no me acuerdo qué número. Es el que si tenemos dos } \\
\text { puntos, podemos encontrar una recta. El axioma } 14 \text { va a } \\
\text { ser el que después represente mecánicamente el } \\
\text { compás. Es el axioma de transporte" }\end{array}$ \\
\hline $\begin{array}{l}\text { Alcance de las } \\
\text { clasificaciones y } \\
\text { denominaciones }(4)\end{array}$ & $\begin{array}{l}\text { "Les voy a hacer otra pregunta: Cuando enunciamos el } \\
\text { teorema nombramos el cuadrado de un segmento, pero } \\
\text { para alguien que no conoce los números tal como lo } \\
\text { hacemos nosotros, ¿qué es el cuadrado de un } \\
\text { segmento?" }\end{array}$ \\
\hline $\begin{array}{l}\text { Alusión al trabajo } \\
\text { matemático (3) }\end{array}$ & $\begin{array}{l}\text { "Fíjense, estuvimos una hora y media para demostrar, } \\
\text { pero así se trabaja en matemática. Uno porque les trae } \\
\text { las cosas cocinadas ya pero hay que sentarse y probar" }\end{array}$ \\
\hline $\begin{array}{l}\text { Vinculación con } \\
\text { ámbito } \\
\text { extramatemático } \\
\text { (3) }\end{array}$ & $\begin{array}{l}\text { "O sea que el área nos da la idea de la dimensión de una } \\
\text { pared, por ejemplo, para saber qué cantidad de pintura } \\
\text { utilizar. Nos da la idea de cómo se distribuye algo no } \\
\text { rígido en una figura plana" }\end{array}$ \\
\hline $\begin{array}{l}\text { Conexión con } \\
\text { futuro desempeño } \\
\text { docente de } \\
\text { estudiantes (2) }\end{array}$ & $\begin{array}{l}\text { "Esta demostración es clásica, la gente del Profesorado } \\
\text { cuando dé clases la pueden dar porque es muy } \\
\text { elemental" }\end{array}$ \\
\hline
\end{tabular}

En la Tabla 4 se evidencia la presencia de la construcción previa a la formalización cada vez que se trabajó algún concepto, lo que subraya la promoción de un proceso gradual al respecto. Las representaciones gráficas 
Tabla 4.

Modalidades y extractos relativos a SCK

\begin{tabular}{|c|c|}
\hline Modalidad & Extracto del relato \\
\hline $\begin{array}{l}\text { Construcción previa a la } \\
\text { formalización (57) }\end{array}$ & $\begin{array}{l}\text { Pregunta qué características tiene y, como muchos } \\
\text { estudiantes mencionan "dos lados congruentes", } \\
\text { dibuja otro trapecio pero ahora rectángulo, } \\
\text { mostrando así que la característica en común es } \\
\text { solamente un par de lados paralelos }\end{array}$ \\
\hline $\begin{array}{l}\text { Representaciones } \\
\text { gráficas (48) }\end{array}$ & $\begin{array}{l}\text { Realiza un dibujo con el cual explica cómo sería la } \\
\text { demostración pero no la escribe }\end{array}$ \\
\hline $\begin{array}{l}\text { Explicación de } \\
\text { estrategias para } \\
\text { demostrar un enunciado } \\
\text { o resolver problemas } \\
\text { (18) }\end{array}$ & $\begin{array}{l}\text { Agrega que siempre que tengan que demostrar } \\
\text { algo, deben pensar con qué herramientas cuentan }\end{array}$ \\
\hline $\begin{array}{l}\text { Justificación de } \\
\text { afirmaciones y } \\
\text { procedimientos (13) }\end{array}$ & $\begin{array}{l}\text { Sin embargo agrega que no es así como lo van a } \\
\text { realizar ellos, sino que lo harán solo con regla y } \\
\text { compás porque estas son las herramientas } \\
\text { permitidas por los axiomas }\end{array}$ \\
\hline $\begin{array}{l}\text { Distintas explicaciones } \\
\text { de una misma idea (9) }\end{array}$ & $\begin{array}{l}\text { Al finalizar, una alumna comenta que no entendió } \\
\text { la parte en que usa el Lema } 4 \text { y le explica de } \\
\text { manera distinta a la anterior }\end{array}$ \\
\hline $\begin{array}{l}\text { Interpretación y análisis } \\
\text { de enunciados (9) }\end{array}$ & $\begin{array}{l}\text { En particular, se detiene en el ítem } 2 \text { y pregunta } \\
\text { qué significa que la imagen sea } \mathrm{R}^{+} \text {. Muchos } \\
\text { estudiantes responden "que es positivo". Aclara } \\
\text { que eso ya lo dice en la parte de "l (PQ) }>0 \text { " }\end{array}$ \\
\hline $\begin{array}{l}\text { Sentido de las } \\
\text { denominaciones y } \\
\text { propiedades }(7)\end{array}$ & $\begin{array}{l}\text { Explica que se conoce como el primer criterio de } \\
\text { congruencia pero, como muy rara vez van a } \\
\text { recordar el orden, simplemente lo van a llamar } \\
\text { LAL (lado-ángulo-lado) }\end{array}$ \\
\hline Trabajo con el error (7) & $\begin{array}{l}\text { Piensan juntos por qué es incorrecto el enunciado. } \\
\text { "Si vivo en A y quiero ir a B, el camino más corto } \\
\text { va a ser ir de A a B que de A a C y de C a B" }\end{array}$ \\
\hline $\begin{array}{l}\text { Direccionamiento de } \\
\text { procedimientos (5) }\end{array}$ & $\begin{array}{l}\text { Al ver que nadie brinda una respuesta } \\
\text { relativamente esperada, les da una "pista" } \\
\text { mencionando que es una combinación del axioma } \\
15 \text { (no del tercer ítem) y la propiedad } 4\end{array}$ \\
\hline $\begin{array}{l}\text { Elección o solicitud de } \\
\text { ejemplos (4) }\end{array}$ & $\begin{array}{l}\text { Con un triángulo obtusángulo para mostrar que no } \\
\text { siempre el pie de la perpendicular está sobre un } \\
\text { lado sino en la recta que lo contiene }\end{array}$ \\
\hline
\end{tabular}


también fueron utilizadas frecuentemente en las clases, sin duda fuertemente impulsadas por el tipo de asignatura en cuestión, y en su mayoría sirvieron de apoyo visual para ideas matemáticas que se estaban gestando. Por lo general, antes de realizar una demostración, se llevó a cabo alguna explicación de estrategias para tal fin, volviendo de este modo a surgir la construcción previa a la formalización.

Tabla 5.

Modalidades y extractos relativos a KCS

\begin{tabular}{|c|c|}
\hline Modalidad & Extracto del relato \\
\hline $\begin{array}{l}\text { Conocimientos previos de } \\
\text { estudiantes (29) }\end{array}$ & $\begin{array}{l}\text { Cuestiona a los alumnos cómo medirían el } \\
\text { pizarrón si tuviesen un centímetro y la mayoría } \\
\text { concuerda en que ponen el centímetro, marcan } \\
\text { hasta dónde llega y en esa marca lo vuelven a } \\
\text { apoyar }\end{array}$ \\
\hline $\begin{array}{l}\text { Niveles de entendimiento } \\
\text { de los estudiantes }(23)\end{array}$ & "Este es un poquito más complicado" \\
\hline $\begin{array}{l}\text { Anticipación a respuesta } \\
\text { (9) }\end{array}$ & $\begin{array}{l}\text { Como los alumnos tardan mucho en medir los } \\
\text { ángulos, dice que después lo hagan en sus } \\
\text { casas, pero que la idea es que les queden dos } \\
\text { ángulos congruentes }\end{array}$ \\
\hline Prevención de errores (9) & $\begin{array}{l}\text { Aclara que dos segmentos congruentes no } \\
\text { necesariamente son iguales como conjuntos de } \\
\text { puntos, pero sí como segmentos }\end{array}$ \\
\hline Autoestima estudiantil (8) & $\begin{array}{l}\text { Asiente, le menciona a la estudiante que está } \\
\text { muy bien }\end{array}$ \\
\hline $\begin{array}{l}\text { Respuesta dada por el } \\
\text { docente }(6)\end{array}$ & $\begin{array}{l}\text { A pesar de la ayuda nadie logra responder } \\
\text { correctamente, por lo que encierra con color el } \\
\text { segundo ítem del axioma } 15\end{array}$ \\
\hline $\begin{array}{l}\text { Indicios de dificultad } \\
\text { estudiantil detectados por } \\
\text { el docente (5) }\end{array}$ & $\begin{array}{l}\text { Parece ser que nota que nadie lo sigue (a mi } \\
\text { criterio, habla mucho y muy seguido) }\end{array}$ \\
\hline
\end{tabular}

Estos procesos promovieron formalizaciones que no se consuman como impuestas, y también estrategias que no se conciban como mágicas. En esta línea además se notaron momentos en que el docente generaba inquietudes acerca de los porqué tanto de las afirmaciones como de los procedimientos, también en cuanto a las denominaciones y propiedades, o cuando promovía 
distintas explicaciones de una misma idea. Atento a las peculiaridades matemáticas en tratamiento, ayudó a interpretar y analizar rigurosamente los enunciados, y también a desglosar lo realizado para aprender a través de eventuales errores, entre otras acciones propias de quien hace uso de la matemática en un ambiente específico de enseñanza.

Como se aprecia en la Tabla 5, los conocimientos previos de los estudiantes fueron muy tenidos en cuenta por el docente al momento de trabajar en sus clases. También realizó, en numerosas oportunidades, comentarios acerca del nivel de entendimiento de los alumnos, presuponiendo la complejidad que tendrían ciertos enunciados o actividades para ellos. Por otro lado, el docente ha intentado prevenir errores, posiblemente en base a experiencias con cursos anteriores, aunque en algunas oportunidades anticipó respuestas a las que estaban aproximándose los estudiantes, o bien, las terminó dando él luego de no haberlas obtenido por parte de ellos. Finalmente supo valorar sus logros y detectar sus dificultades, favoreciendo la autoestima del estudiantado y la comunicación en el aula.

Tabla 6.

Modalidades y extractos relativos a KCT

\begin{tabular}{|c|c|}
\hline Modalidad & Extracto del relato \\
\hline $\begin{array}{l}\text { Comentario sobre } \\
\text { desarrollo de clases (35) }\end{array}$ & $\begin{array}{l}\text { Agrega que van a enunciar todos los criterios } \\
\text { juntos, que después los demuestran y si hacen } \\
\text { tiempo resuelven algún ejercicio }\end{array}$ \\
\hline $\begin{array}{l}\text { Formulación de una nueva } \\
\text { pregunta }(27)\end{array}$ & $\begin{array}{l}\text { Muchos alumnos responden " } \pi r^{2} \text {. Agrega "¿Y } \\
\text { qué es } \pi \text { ?" }\end{array}$ \\
\hline $\begin{array}{l}\text { Indagación sobre } \\
\text { entendimiento (16) }\end{array}$ & $\begin{array}{l}\text { Sin embargo vuelve a preguntar si se entendió el } \\
\text { axioma y, como nadie responde, dice: "Levante } \\
\text { la mano el que no entendió. ¿Todo el mundo } \\
\text { entendió?" }\end{array}$ \\
\hline $\begin{array}{l}\text { Conclusión y cierre de } \\
\text { idea (14) }\end{array}$ & $\begin{array}{l}\text { Concluyendo nuevamente que "no genera } \\
\text { criterio de congruencia y que vamos a necesitar } \\
\text { tres datos como mínimo" }\end{array}$ \\
\hline $\begin{array}{l}\text { Explicación a partir de } \\
\text { respuesta de estudiante } \\
\text { (12) }\end{array}$ & $\begin{array}{l}\text { Un alumno exclama: “'Un lado!”, a lo que } \\
\text { responde: "Bien, cualquier lado. La base de un } \\
\text { triángulo es un lado y según el lado que elija voy } \\
\text { a tener cierta altura” }\end{array}$ \\
\hline
\end{tabular}


Tabla 6. (.../...)

\begin{tabular}{ll}
\hline Modalidad & Extracto del relato \\
\hline Repaso de contenidos (10) & $\begin{array}{l}\text { Continúa realizando un repaso de lo dado en la } \\
\text { última clase de teoría }\end{array}$ \\
$\begin{array}{l}\text { Explicación a partir de } \\
\text { pregunta de estudiante (9) }\end{array}$ & $\begin{array}{l}\text { Una alumna dice que no entiende la pregunta, } \\
\text { por lo que procede a explicarla y entre todos se } \\
\text { dan cuenta que sí es posible utilizar el teorema } \\
\text { Le solicita a una alumna que le cuente qué dice } \\
\text { el teorema. La estudiante accede }\end{array}$ \\
$\begin{array}{l}\text { pportes estudiantiles } \\
\text { cuenta (8) }\end{array}$ & $\begin{array}{l}\text { Eso intencional de colores } \\
\text { (5) }\end{array}$ \\
\hline
\end{tabular}

En la Tabla 6 se muestra que en reiteradas ocasiones el docente realizó comentarios sobre el desarrollo de sus clases, dando la oportunidad a los estudiantes de organizarse. La formulación de una nueva pregunta estuvo muy presente, mayoritariamente acompañando la construcción previa a la formalización mencionada en el SCK. Las frecuencias de aparición de algunas modalidades denotan que el docente tuvo en cuenta los comentarios, aportes y respuestas de los estudiantes, permitiendo que sean partícipes de las clases. También se esforzó por hacer conclusiones y cierres parciales de las ideas, sin dejar razonamientos aislados o descontextualizados. En momentos puntuales empleó fibrón de algún color distinto al negro para resaltar algo.

Tabla 7.

Modalidades y extractos relativos a KCC

\begin{tabular}{ll}
\hline Modalidad & Extracto del relato \\
\hline $\begin{array}{l}\text { Apoyo en apunte de la } \\
\text { asignatura (25) }\end{array}$ & $\begin{array}{l}\text { Comenta que lo va a explicar rápido } \\
\text { primero, que la idea es que lo entiendan y } \\
\text { después lo pueden leer bien del apunte }\end{array}$ \\
$\begin{array}{l}\text { Articulación teoría-práctica } \\
\text { (18) }\end{array}$ & $\begin{array}{l}\text { Recuerda el ejercicio 6 de la sección 2.1 } \\
\text { para concluir que AB=DC y AD=BC }\end{array}$ \\
$\begin{array}{l}\text { Articulación vertical en la } \\
\text { misma asignatura (11) }\end{array}$ & $\begin{array}{l}\text { Recuerda cómo hacían para medir } \\
\text { segmentos y lo compara con la medición de } \\
\text { áreas }\end{array}$ \\
\hline
\end{tabular}


Tabla 7. (.../...)

\begin{tabular}{ll}
\hline Modalidad & Extracto del relato \\
\hline $\begin{array}{l}\text { Articulación contenidos- } \\
\text { tiempo (4) }\end{array}$ & $\begin{array}{l}\text { "En realidad si tuviésemos tiempo les diría } \\
\text { que agarren un triángulo, recorten los } \\
\text { ángulos y los peguen todos consecutivos" }\end{array}$ \\
$\begin{array}{l}\text { Articulaciones horizontal y } \\
\text { vertical en el marco de la }\end{array}$ & $\begin{array}{l}\text { Teorema de Pitágoras, haciendo referencia } \\
\text { a lo dado en Álgebra en la parte de Lógica }\end{array}$ \\
carrera (4) & $\begin{array}{l}\text { Comenta que la demostración original de } \\
\text { Materiales de consulta y }\end{array}$ \\
estudio (4) & $\begin{array}{l}\text { Euclides la pueden buscar en Internet } \\
\text { Lo lee y dice que "este enunciado está en la } \\
\text { Articulación con nivel }\end{array}$ \\
secundario de educación (3) clásica como se da en la secundaria. \\
& $\begin{array}{l}\text { Nosotros vamos a hacer un análisis más } \\
\text { crítico y vamos a ver que en realidad es más } \\
\text { simple" }\end{array}$ \\
\hline
\end{tabular}

En algunas ocasiones, tal como se observa en la Tabla 7, el docente contó con el apunte de la asignatura principalmente como apoyo para el estudio de los mismos estudiantes, incentivando su lectura como complemento a las clases. Además es posible notar que, en su mayoría, los contenidos no fueron presentados aislados, sino que se realizaron articulaciones en la propia asignatura (de forma vertical y con la práctica), en la carrera y con el nivel secundario de educación.

\section{Discusión}

A partir del análisis efectuado, fue posible advertir algunas regularidades, como por ejemplo, que en las clases se desarrolló una estructura recurrente de la forma repaso-desarrollo-cierre. Entre los dos primeros dominios más activados en cada clase siempre se encontró el SCK acompañado, por lo general, del KCT (aunque hay excepciones). El primero de ellos solía aparecer en el desarrollo de la clase mientras que, el segundo, al inicio de las mismas. Esto podría dar cuenta de que, al trabajar con los contenidos nuevos, el docente puso en juego cuestiones propias de la profesión, tal como la construcción previa a la formalización, que demuestran cierta comprensión de los conceptos que permite presentarlos de distintas 
maneras, mediante variedad de registros, en diversos contextos. Por otro lado, al comenzar las clases, se evidencian en mayor medida los comentarios sobre el desarrollo de las mismas y el repaso de contenidos, logrando de este modo una "puesta en situación" de los estudiantes frente a la asignatura.

En aquellos momentos donde se introdujo algún concepto, las modalidades con mayor presencia fueron Contexto histórico, Construcción previa a la formalización, Conocimientos previos de estudiantes, Formulación de una nueva pregunta, Comentario sobre desarrollo de clases y Representaciones gráficas. Es decir, en general cada concepto se construyó en conjunto con los alumnos, mostrando su contexto y relación con los demás.

En el cierre de las clases fue habitual hacer referencia a los Niveles de entendimiento de los estudiantes, al Comentario sobre desarrollo de clases, al Apoyo en el apunte de la asignatura y a la Articulación, ya sea teoríapráctica o con la misma asignatura. Con esto se evidencia el interés del docente en que los alumnos comprendan lo trabajado y lo puedan utilizar para resolver las actividades propuestas, incentivando la lectura individual del apunte a modo de refuerzo.

Además, algunas modalidades estuvieron acompañadas por otras, como por ejemplo, Representaciones gráficas y Construcción previa a la formalización, o bien, Explicación a partir de respuesta de estudiante con Formulación de una nueva pregunta, y Apoyo en apunte de la asignatura con Articulación teoría-práctica. También, si bien hubo excepciones, en la mayoría de los casos la Explicación de procedimientos fue seguida por su respectiva Verificación y Justificación. Estas cuestiones resaltadas pueden observarse en el esquema lógico-conceptual que se presenta en la Fig. 3.

Entre las oportunidades para la construcción de conocimiento identificadas, se destaca el repaso de contenidos previos al inicio de cada clase, como una actividad observada con recurrencia. Esta modalidad del dominio KCT ayuda tanto a los alumnos como al docente a ponerse en situación, favoreciendo de este modo la comprensión y construcción de los conceptos posteriores.

La articulación de contenidos atiende a una modalidad detectada en el dominio KCC, mediante la cual el docente debe relacionar lo nuevo con lo que supone que los estudiantes aprendieron en la secundaria, o bien, con lo trabajado previamente ya sea en esta $u$ otra materia. De esta manera se 
154 Schaefer \& Sgreccia - Enseñanza de la geometría sintética
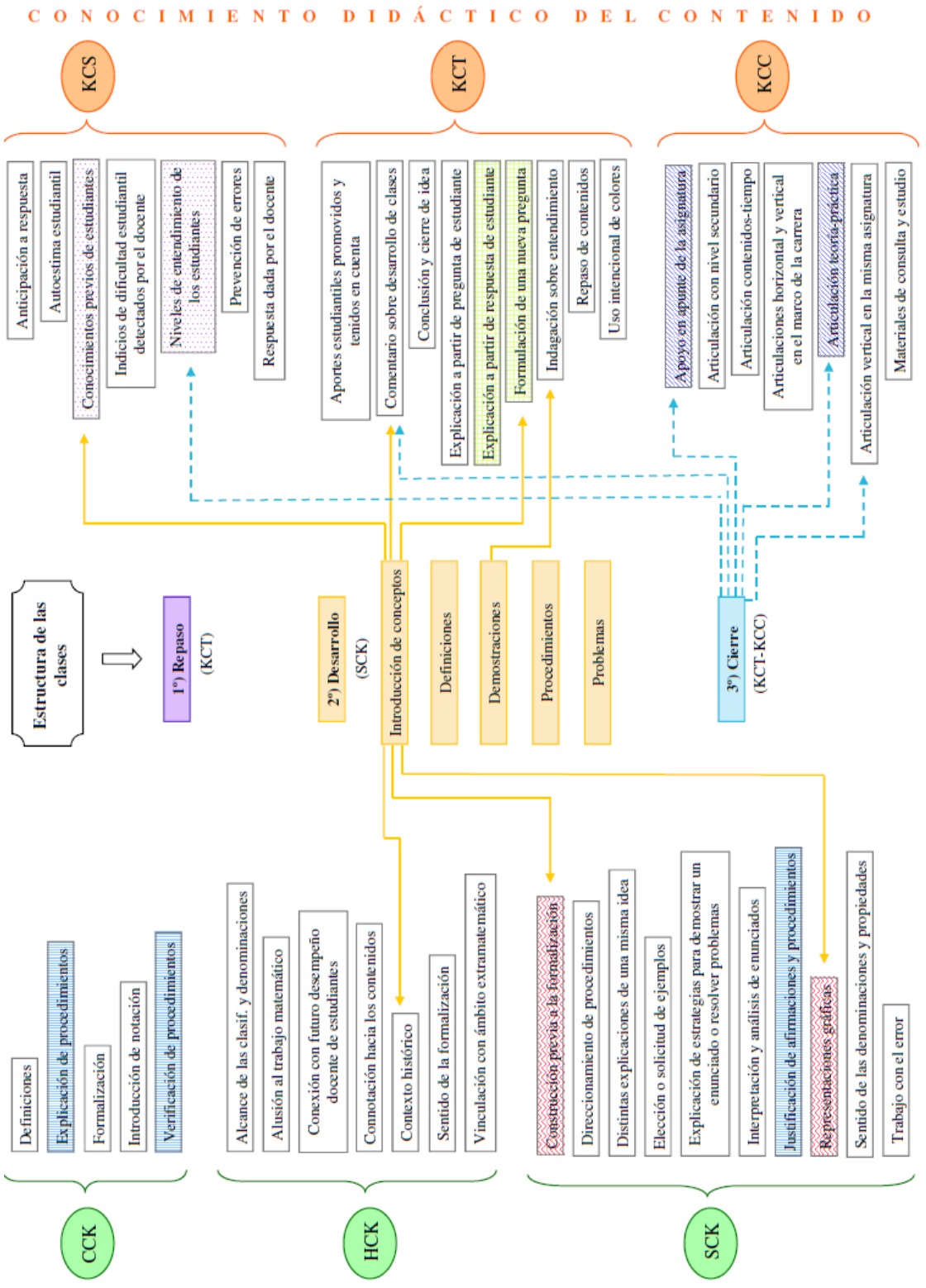

Figura 3. Esquema lógico-conceptual de las clases observadas 
colabora con mostrar la matemática de manera integrada, evitando su fragmentación en conceptos aislados.

En Geometría las representaciones gráficas juegan un papel importante, pero no son la única herramienta para construir los conceptos. La formulación de preguntas orientadoras, cuestión remarcada en el dominio $\mathrm{KCT}$, puede transformarse en un instrumento muy útil para tal fin, pero para ello se debe dar lugar a la participación estudiantil. Se sugiere que todo profesor, decidido a guiar sus clases con preguntas, debe escuchar a sus alumnos, esperarlos, darles el tiempo necesario para que comprendan la pregunta y elaboren una respuesta.

En cuanto al error como recurso de enseñanza y aprendizaje (modalidad distinguida en el dominio KCS), el docente puede preverlos tanto a partir de su propia experiencia con otros grupos, como así también de las dificultades con que se encontró cuando aprendió el concepto, o de lecturas realizadas de artículos de investigación en el área. Estas suposiciones que realiza en cuanto a los posibles errores estudiantiles, debería aprovecharlas, por ejemplo, planteando intencionalmente situaciones donde suponga que el alumno tendrá alguna dificultad, incentivando el análisis de la misma en conjunto con la clase. Por otro lado, frente al error de un estudiante, el docente debe ser muy cuidadoso con las palabras que utiliza y con los modos de remarcar el mismo. Es importante que se trabajen junto al grupo no solo los errores de los alumnos, sino también los eventuales del profesor, por ejemplo, analizando paso a paso qué es lo incorrecto, qué otras situaciones podrían haber surgido, cuál sería una respuesta conveniente y por qué.

Respecto al conocimiento de la disciplina, es necesario que el docente cuente con herramientas más avanzadas -con respecto a las que emplea de manera directa en el curso- para poder manipular con mayor libertad el contenido y abordarlo de la mejor manera posible. Pero "saber más" no coincide con "hablar más"; el profesor deber ser consciente que algunas cuestiones podrían confundir a los estudiantes. Por ello es fundamental que entienda la necesidad de "callar" ciertas cosas, de reservarlas para cuando los alumnos hayan comprendido el concepto principal que se está trabajando.

Además de detectar oportunidades, en el estudio realizado se observaron algunas limitaciones de la enseñanza que pueden repercutir en los aprendizajes. Un ejemplo de ello es el escaso uso del material concreto en 
la educación superior, a pesar de ser una herramienta que ayuda a los alumnos en la manipulación y visualización de los conceptos geométricos. En dicho nivel se suele considerar que el material concreto es una manera de perder el tiempo; se cree, entonces, que existe una debilidad significativa en el proceso de formación de profesores, la cual debería ser estudiada en mayor profundidad.

Posiblemente, en el nivel superior, el uso de colores variados en el pizarrón parezca algo muy simple (cuestión observada y remarcada en el $\mathrm{KCT}$ ). Sin embargo es un recurso que colabora con la visualización y posterior comprensión de los contenidos, principalmente en Geometría. El docente debería tenerlos en cuenta, por ejemplo, para diferenciar la hipótesis y la tesis en un enunciado, o para remarcar conceptos en un mismo dibujo. También, en términos de colaborar con la visualización, podría ser útil la utilización de algún software geométrico.

Otra limitación remarcada es el abordaje del lenguaje matemático. Muchas veces será conveniente partir del vocabulario habitual de los estudiantes para, de a poco, pulirlo y llegar a una definición formal. Algo similar se podría tener en cuenta con los procedimientos, transformándolos con la pregunta del docente en un axioma o un teorema. Los alumnos de primer año posiblemente no hayan enfrentado antes la escritura matemática. Por ello es necesario que el docente realice aclaraciones sobre la notación (modalidad destaca en el CCK), que enseñe a escribir y a leer el nuevo lenguaje; para ello puede ser de utilidad expresar coloquialmente aquellos enunciados simbólicos que se trabajan por primera vez.

\section{Conclusiones}

Al retomar la primera conjetura planteada (la versión de la Geometría Sintética estudiada en el Profesorado en Matemática dista considerablemente de una versión escolar relativamente propicia de procesos constructivos en la escuela secundaria), se puede concluir que durante las clases observadas de Geometría I se ha propiciado la construcción de conceptos, analizando su origen, sus "porqué", conectando los contenidos y, de este modo, favoreciendo la futura enseñanza de los mismos. Es decir, si bien en las clases se podría realizar mayor cantidad de relaciones con el nivel secundario, la distancia mencionada en la conjetura 
propuesta no es tan grande como se suponía. Sin embargo se cree que este acercamiento no se hace explícito a los futuros profesores en las clases; ¿será que el mismo es llevado a cabo en alguna otra asignatura correspondiente a la práctica docente?, ¿o simplemente es un trabajo que debe realizar cada uno de los estudiantes, de manera individual y por su propia cuenta? Este es uno de los puntos que se considera se debería seguir estudiando desde la investigación e insistiendo desde la formación docente (Godino et al, 2016).

Respecto a la segunda conjetura señalada (el conocimiento especializado del contenido de Geometría Sintética, requerido para la enseñanza, queda relegado a la construcción, individual y sin vigilancia epistémica, del estudiante), se cree que en las clases observadas se evidencia una gran riqueza en las modalidades surgidas en torno al conocimiento especializado del contenido, siendo uno de los subdominios que agrupa la mayor cantidad de modalidades (10 en total, las cuales comprenden cuestiones referidas a la construcción de conceptos, su representación gráfica, la utilización de ejemplos, las estrategias para resolver o demostrar enunciados y la interpretación de los mismos). Por ello, se considera que el conocimiento especializado del contenido no queda relegado totalmente a la construcción propia del estudiante, sino que el docente colabora en la misma y otorga diversas herramientas para que el futuro profesor se apropie de este tipo de conocimiento necesario para llevar a cabo su trabajo.

Por otro lado, el profesor a cargo de la asignatura evidenció contar con un importante conocimiento acerca de la historia de la disciplina y disfrutaba transmitirlo. Asimismo quedan inquietudes acerca de su potencial didáctico en las clases (De Gamboa et al., 2015). Se ha tenido indicios de que el docente considera que el conocimiento debe ser construido por los alumnos, razón por la cual incentivó la participación de los mismos en las clases. Permitió que los estudiantes conjeturen, pregunten, reflexionen, relacionen conceptos, trabajen con el error. Se lo notó muy comprometido con la preparación de sus clases, demostrando interés en proporcionar situaciones de aprendizaje en el aula de la manera más clara y adecuada posible. Mencionó conceptos propios de la escuela secundaria, tomándolos como conocimiento previo de los alumnos, relacionando ambos niveles. Procuró repasar las explicaciones la cantidad de veces que sea necesario, de distintas maneras, a veces realizando analogías con la vida cotidiana o utilizando software geométrico. A pesar 
de no haber recibido una formación pedagógica específica, demostró tener sus propias concepciones sobre la enseñanza y el aprendizaje, basadas en la experiencia y la reflexión. Sin embargo, en algunas ocasiones fue impaciente frente a las respuestas de los alumnos, cuestión que intentó mejorar con el tiempo. Estas respuestas, al comienzo de las observaciones, eran breves, poco concisas y solo surgían si el docente preguntaba. Con el correr de los días, los alumnos comenzaron a participar en mayor medida, a cuestionar y a trabajar, generándose cesión y traspaso progresivos de la responsabilidad y control en el aprendizaje, en términos de Coll et al. (1992). La relación entre ellos era muy buena, lo cual permitió un cómodo clima de trabajo grupal.

Las cuestiones presentadas pretenden promover la reflexión sobre la propia práctica, la cual debería acompañar a los docentes durante toda la carrera, y teniendo en cuenta el caso de la asignatura en estudio, dirigida a profesores en formación, resulta fructífero que también se transmita a los alumnos del Profesorado para que ellos también lo tengan en cuenta en su futura práctica, como señalan Sgreccia y Massa (2012) y Yang (2012).

Finalmente, se destaca que lo presentado en este artículo, tanto las modalidades emergentes como las limitaciones y oportunidades para la construcción de conocimiento geométrico reconocidas, contribuye a conocer acerca de las prácticas de enseñanza para la construcción de conocimientos de Geometría Sintética en la formación inicial de profesores en Matemática. También puede resultar de utilidad para abordar el análisis de la enseñanza y el aprendizaje de otras temáticas, asignaturas o áreas de la Matemática. Incluso puede ser tomado para estudiar el caso de otros Profesorados en Matemática en el área de Geometría Sintética.

\section{Bibliografía}

Acosta, M., Mejía, C. y Rodríguez, C. (2013). Lugares geométricos en la solución de un problema de construcción: presentación de una posible técnica de una praxeología de geometría dinámica. Educación Matemática, 25(2), 141-160.

Atiyah, M. (2001). Mathematics in 20th Century: Geometry vs Algebra. Mathematics Today, 37(2), 47-49. 
Ball, D. (1988). Knowledge and reasoning in mathematical pedagogy: Examining what prospective teachers bring to teacher education. Tesis de Doctorado. East Lansing: Michigan State University. Ball, D., Thames, M. y Phelps, G. (2008). Content Knowledge for Teaching. What Makes It Special? Journal of Teacher Education, 59(5), 389-407. Doi: 10.1177/0022487108324554

Barrantes, M. y Blanco, L. (2005). Análisis de las concepciones de los profesores en formación sobre la enseñanza y aprendizaje de la geometría. Números, 62, 33-44.

Borba, M. (2006). Diversidade de questões em formação de professores de matemática. En M. Borba (Coord.). Tendências Internacionais em Formação de Professores de Matemática (pp.9-26). Belo Horizonte: Autêntica.

Ciccioli, V. y Sgreccia, N. (2017). Formación en geometría analítica para futuros profesores. Estudio de caso basado en el MKT. Educación Matemática, 29(1), 141-170. Doi: 10.24844/em2901.06

Coll, C., Colomina, R., Onrubia, J. y Rochera, M. (1992). Actividad conjunta y habla: una aproximación al estudio de los mecanismos de influencia educativa. Infancia y aprendizaje, 59-60, 189-232. Doi: 10.1080/02103702.1992.10822356

Consejo Interuniversitario Nacional (2013). Estándares para la Acreditación de las carreras de Profesorado Universitario en Matemática. Retrieved from: http://www.cin.edu.ar/descargas/asuntosacademicos/18-12$\%$ 20Subcom.\%20Profesorados\%20$\%$ 20Estandares\%20MATEMATICA.doc

Corica, A. y Marin, E. (2014). Actividad de estudio e investigación para la enseñanza de nociones de geometría. Números, 85, 91-114.

De Gamboa, G., Badillo, E. y Ribeiro, M. (2015). El horizonte matemático en el conocimiento para la enseñanza del profesor: geometría y medida en educación primaria. PNA, 10(1), 1-24.

Escudero, D., Carrillo, J. y Flores, E. (2015). El conocimiento especializado del profesor de matemáticas detectado en la resolución del problema de las cuerdas. PNA, 10(1), 53-77.

García, M. (2005). La formación de profesores de matemáticas. Un campo de estudio y preocupación. Educación Matemática, 17(2), 153-166. 
160 Schaefer \& Sgreccia - Enseñanza de la geometría sintética

Gascón, J. (2002). Geometría sintética en la ESO y analítica en el Bachillerato. ¿Dos mundos completamente separados? Suma, 39, 1325.

Godino, J.D., Gonzato, M., Contreras, A., Estepa, A. y Díaz-Batanero, C. (2016). Evaluación de Conocimientos Didáctico-Matemáticos sobre Visualización de Objetos Tridimensionales en Futuros Profesores de Educación Primaria. REDIMAT, 5(3), 235-262. Doi:

10.4471/redimat.2016.1984

Gutiérrez, A. (2010). Introducción al Seminario I sobre Enseñanza y Aprendizaje de la Geometría. En M. Moreno, A. Estrada, J. Carrillo y T. Sierra (Eds.). Investigación en Educación Matemática XIV (pp.1719). Lleida: Sociedad Española de Investigación en Educación Matemática.

Ministerio de Educación (2010). Proyecto de mejora para la formación inicial de profesores para el nivel secundario - Matemática (pp.118179). Buenos Aires: Ministerio de Educación.

Santana, N. y Climent, N. (2015). Conocimiento Especializado del Profesor para la utilización de GeoGebra en el Aula de Matemáticas. Números, 88, 75-91.

Sgreccia, N. y Massa, M. (2012). 'Conocimiento especializado del contenido' de estudiantes para profesor y docentes noveles de matemáticas. El caso de los cuerpos geométricos. Educación Matemática, 24(3), 33-66.

Taylan, R.D. y Da Ponte, J.P. (2016). Investigating pedagogical content knowledge-in-action. REDIMAT, 5(3), 212-234. Doi: 10.17583/redimat.2016.2227

Yang, K-J. (2012). How do elementary preservice teachers form beliefs and attitudes toward geometry learning? Implications for teacher preparation programs. REDIMAT, 1(2), 194-213. Doi: 10.4471/redimat.2012.10 
Lucía Inés Schaefer es profesora de matemáticas, de la Universidad Nacional de Rosario, Argentina.

Natalia Fátima Sgreccia es profesora de enseñanza media y superior de matemáticas y magíster en didácticas específicas con mención en el área de matemática, y doctora en Humanidades y Artes con mención en Ciencias de la Educación, de la Universidad Nacional de Rosario, Argentina.

Dirección de contacto: La correspondencia directa sobre este artículo debe enviarse al autor. Dirección Postal: Universidad de Rosario. Facultad de Ciencias Exactas, Ingeniería y Agrimensura. Av/ Pellegrini, 250, Rosario, Santa Fe, Argentina. Email: lucias@fceia.unr.edu.ar 